\title{
Alteration of brain insulin and leptin signaling promotes energy homeostasis impairment and neurodegenerative diseases
}

\author{
Mohammed TAOUIS \\ Laboratoire de neuroendocrinologie \\ moléculaire de la prise alimentaire, \\ Centre de neurosciences Paris Sud \\ (CNPS), \\ UMR 8195 CNRS Université Paris Sud \\ $X I$, \\ IBAIC, \\ Bât. 447 , \\ 91405 Orsay cedex, \\ France \\ <mohammed.taouis@u-psud.fr>
}

\begin{abstract}
The central nervous system (CNS) controls vital functions, by efficiently coordinating peripheral and central cascades of signals and networks in a coordinated manner. Historically, the brain was considered to be an insulin-insensitive tissue. But, new findings demonstrating that insulin is present in different regions of the mammalian brain, in particular the hypothalamus and the hippocampus. Insulin acts through specific receptors and dialogues with numerous peptides, neurotransmitters and adipokines such as leptin. The cross-talk between leptin and insulin signaling pathways at the hypothalamic level is clearly involved in the control of energy homeostasis. Both hormones are anorexigenic through their action on hypothalamic arcuate nucleus by inducing the expression of anorexigenic neuropetides such as POMC (pro-opiomelanocortin, the precursor of $\alpha M S H$ ) and reducing the expression of orexigenic neuropeptide such as NPY (Neuropeptide Y). Central defect of insulin and leptin signaling predispose to obesity (leptin-resistant state) and type-2 diabetes (insulin resistant state). Obesity and type-2 diabetes are associated to deep alterations in energy homeostasis control but also to other alterations of CNS functions as the predisposition to neurodegenerative diseases such as Alzheimer's disease (AD). AD is a neurodegenerative disorder characterized by distinct hallmarks within the brain. Postmortem observation of $A D$ brains showed the presence of parenchymal plaques due to the accumulation of the amyloid beta (AB) peptide and neurofibrillary tangles. These accumulations result from the hyperphosphorylation of tau (a mictrotubule-interacting protein). Both insulin and leptin have been described to modulate tau phosphorylation and therefore in leptin and insulin resistant states may contribute to $A D$. The concentrations of leptin and insulin cerebrospinal fluid are decreased type 2 diabetes and obese patients. In addition, the concentration of insulin in the cerebrospinal fluid of $A D$ patients is diminished.

Taken together, these data clearly links deficiency of leptin and insulin signaling to both alterations of energy homeostasis control and predisposition to AD. Furthermore, environment changes leading to insulin and leptin-resistance may promote these defects, such as high fat diet.
\end{abstract}

Key words: central nervous system, homeostasis, insulin, leptin, neurodegenerative diseases

the associated metabolic diseases such as type 2 diabetes, hypertension and cardiovascular diseases are considered as major public health problem all around the world. Furthermore, obesity and type 2 diabetes are also linked to other alterations of CNS functions as the predisposition to neurodegenerative diseases such as Alzheimer's disease (AD) (Elias et al., 2005; Paz-Filho et al., 2010).
Thus, a better understanding of the mechanisms involved in the hypothalamic control of food intake is essential at both basic science and clinical levels concerning obesity, type 2 diabetes and AD.

The hypothalamus is the main brain location controlling energy homeostasis. It receives peripheral signals such as one of the major causes contributing the prevalence of obesity. Obesity and

To cite this article: Taouis M. Alteration of brain insulin and leptin signaling promotes energy homeostasis impairment and neurodegenerative diseases. OCL 2011; 18(5): 251-254. doi : 10.1684/ocl.2011.0405 
changes in circulating levels of various hormones and nutrients, and integrates these signals to respond to the energy body requirements which generally match the energy expenditure. There are tremendous number of publications concerning the role of the hypothalamus in controlling energy homeostasis through changes in circulating levels of hormones such as leptin and insulin that are considered as adiposity signals, and also of nutrients such as glucose, amino and fatty acids (Schwartz and Porte Jr, 2005). The alteration of these signals contributes to the impairment of hypothalamic energy homeostasis control, leading to the onset of obesity which is currently considered as a worldwide health epidemic (Hofbauer, 2003). However, the exact mechanisms concerned, are rather unexplored. The field is even more under investigated in stages preceding the onset of metabolic diseases and AD.

In this review we will focus on the role of two major hormones involved in the energy homeostasis control and that have been recently also involved in AD: insulin and leptin.

\section{Role insulin and leptin in the control of energy homeostasis}

Leptin and insulin are regulators of energy homeostasis and both reduce food intake through the activation of their respective receptors in the hypothalamus and specifically in arcuate nucleus (Szanto and Kahn, 2000; Berthou et al., 2011; Benomar et al., 2005). Changes in leptinemia and insulinemia inform the hypothalamus of the energy status of the whole body.

Leptin, an adipokine, is an important regulator of energy homeostasis. Acting through its hypothalamic receptors, ObrB long form, leptin reduces food intake by up regulating anorexic neuropeptides (POMC) and reducing orexic neuropeptides (NPY, AgRP). Leptin receptor signaling pathways are well documented and involve several signal molecules such as JAK2/STAT-3, MAP kinases and IRS/PI3 kinase (Friedman, 1998). However, the hope brought by the discovery of leptin has been rapidly followed by a great deception, since leptin treatment seemed to be inefficient to correct obesity. In most cases of obesity, patients show a sustained hyperphagia despite high plasma leptin levels, suggesting a leptin resistant state.

Insulin pancreatic secretion is under metabolic, endocrine and neural controls. These factors have a direct impact on circulating insulin levels and on the transport of blood insulin in the brain. As in peripheral tissues, insulin acts in the brain through insulin receptors (IR) activating several signaling pathways such as IRS/PI3K, MAP kinase and JAK2/ STAT-3. While the effect of leptin in the hypothalamus is well documented, the role of insulin has been suggested by the observation that insulin-deficient animals are hyperphagic and the administration of insulin in the third ventricle normalizes their food intake and body weight (Elias et al., 2005; Gerozissis, 2004). In the hypothalamus, insulin as leptin acts on pro-opiomelanocortin (POMC) neurons activating the secretion of an anorexic neuropeptide, aMSH (amelanocyte-stimulating hormone), and on NPY/AGRP neurons by inhibiting the expression of the orexic neuropeptide NPY (Elias et al., 2005). It is noteworthy that both central leptin and insulin resistances can lead to hyperphagia, increased plasma insulin and leptin concentrations, and changes in energy balance and fat mass (Niswender et al., 2004).

Evidence accumulated over the last decade concerning the possible cross-

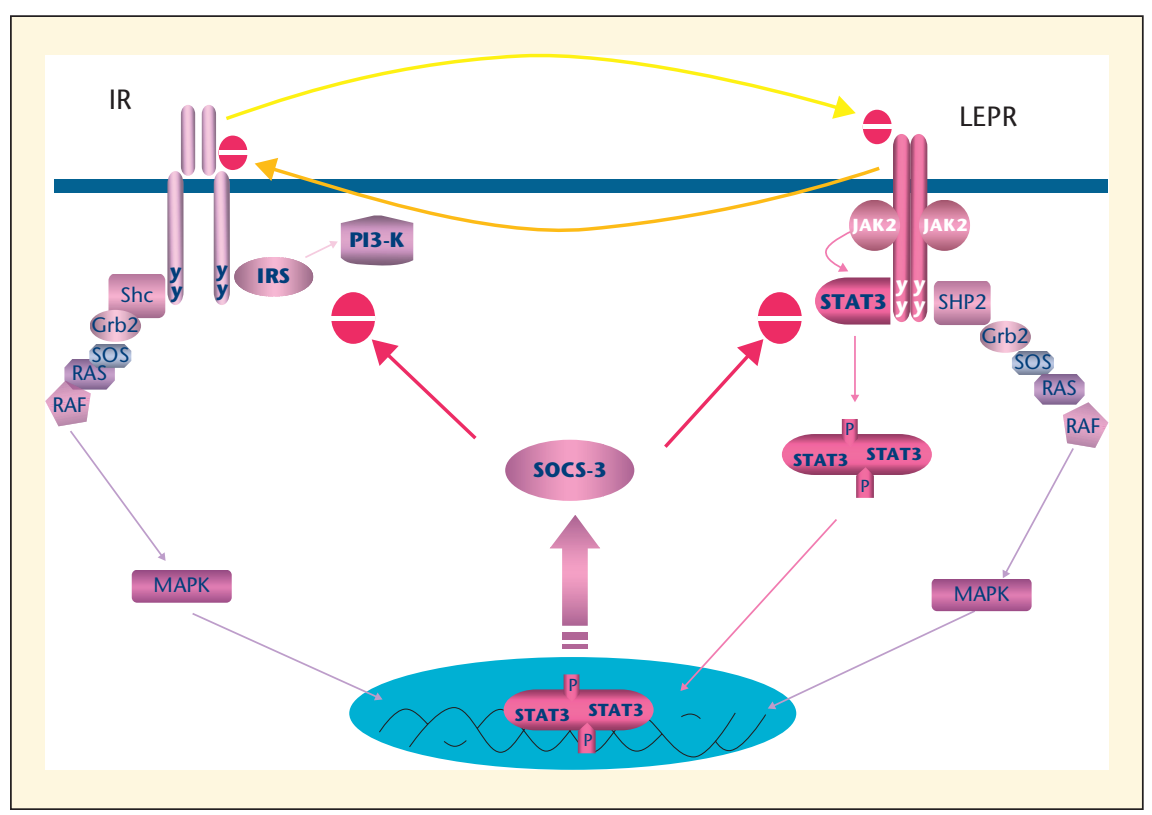

Figure 1. Insulin and leptin cross-desensitization. talk between insulin and leptin sensitivity at both central and peripheral levels which may possibly link the insulin and leptin resistance, and consequently lead to obesity and type-2 diabetes (figure 1). Studies performed in hepatic or neuroinsulin share several signaling pathways such as JAK-2/STAT-3, IRS/PI3kinase/Akt and MAP kinase (Szanto and Kahn, 2000; Carvalheira et al., 2005; Benomar et al., 2009). Furthermore, leptin treatment of leptin deficient ob/ob mice reduces blood insulin and glucose concentrations suggesting improved insulin sensitivity (Pelleymounter et al., 1995). This treatment also improved glucose utilization and insulin sensitivity in liver and muscle in normal rodents (Berthou et al., 2011). Leptin also increases or insulin-resistant rats. Interestingly, a chronic central leptin treatment increases insulin-stimulated muscle and brown adipose tissue glucose utilization (Cusin et al., 1998). It has been also reported that leptin directly modulates insulin secretion by reducing insulin mRNA expression and insulin secretion in pancreatic $\beta$ cells (Berthou et al., 2011; Ott et al., 1996).

Furthermore, the cross-talk between insulin and leptin signaling may also involve a negative regulator, Phosphotyrosine Phosphatase 1B (PTP-1B). PTP$1 \mathrm{~B}$ is able to dephosphorylate several nal cells reported that both leptin and peripheral insulin sensitivity in diabetic 
components of leptin and insulin signaling pathways such as: IRS1/2, JAK2 (Benomar et al., 2009)(12). We have previously demonstrated a cross-desensitization between leptin and insulin signaling pathways involving PTP-1B in human neuroblastoma cell line (Benomar et al., 2005).

Taken together, these data suggest that the augmentation of circulating leptin subsequently to a body weight gain may contribute to progressive onset of insulin-resistance at both central and peripheral level.

\section{Alzheimer's disease linked to insulin and leptin signaling impairment}

Epidemiological studies demonstrated that $A D$ is more common in patient with type-2 diabetes (Ott et al., 1996). Furthermore, the persistent hyperglycemia seems to play a crucial role in cerebral dysfunctions (Reaven et al., 1990). These studies and many others suggest that insulin resistance may contribute to neurodegenerative disease such as AD.

Recently, crucial extra-hypothalamic functions have been attributed to leptin on brain structure (Paz-Filho et al., 2010). Leptin is involved in several functions beside its impact on regulation of energy homeostasis such as neurogenesis, axon growth, synapto- genesis events that occur early in life (Boret, 2010). Furthermore, a neuroprotective role has been also attributed to leptin as mirrored by the inhibition of apoptose and improving cell survival by protecting against oxidative stress (Morrison, 2009). Interestingly, low circulating leptin have been associated to a direct cause of cognitive impairment observed in AD.

Thus, the adipoinsular axis, consisting of insulin and leptin, is crucial for the regulation of brain functions (3), linking then leptin, obesity, type- 2 diabetes and AD. Consequently, brain insulin and leptin resistance are risk factors for $A D$ and dementia (figure 2). In fact, insulin resistance and low insulin levels in the central nervous system would lead to the accumulation of beta-amyloid (the pathologic hallmark of AD) and neurofibrillary tangles (Takeda et al., 2010). These accumulations result from the hyperphosphorylation of Tau (a microtubule-interacting protein). Both insulin and leptin have been described to modulate tau phosphorylation and therefore insulin and leptin resistance states may contribute to the hyperphosphorylation of tau. In fact in normal state, insulin and leptin through the PI3-kinase/Akt signaling pathway inhibit phosphorylation GSK-3 and consequently reducing tau phosphorylation (Liu et al., 2011). Diabetes also causes vascular remodeling leading to increased RAGE (receptor for advanced glycation endproducts) expression leading to cerebrovascular amyloid angiopathy as observed in AD (Sato et al., 2011). Furthermore, an enzyme involved in the catabolism of insulin, insulin-degrading enzyme (IDE), in normal subject has been found to degrade beta-amyloid protein in neuronal and microglia cells in order to eliminate the neurotoxic effect of beta-amyloid. However, during the onset of insulin resistance which is characterized by hyperinsulinemia, IDE is most-likely saturated by insulin and is not efficiently involved in beta-amyloid degradation favoring then its accumulation as in AD (Farris et al., 2003).

If we extrapolate the cross-talk between insulin and leptin signaling pathways described for the energy homeostasis to $A D$, hyperleptinemia will be considered as a serious risk factor for AD by altering insulin responsiveness and signaling at the neuronal level.

\section{Conclusion}

The cross talk between insulin and leptin signaling pathways in the brain may have a crucial role for both energy homeostasis and cognition. During the onset of obesity that is characterized by increased plasma leptin levels and leptin resistance, insulin resistance is promoted at the central level. These changes deeply affect both leptin and insulin signaling by a clear defect of PI 3-kinase/Akt pathway leading to the impairment of food intake regulation and the increase of tau phosphorylation leading to the accumulation of betaamyloid favoring then AD.

\section{REFERENCES}

Benomar Y, Roy AF, Aubourg A, Djiane J, Taouis M. Cross-down regulation of leptin and insulin receptor expression and signaling in a human neuronal cell line. Biochem J 2005; 388: 929-39.

Benomar Y, Berthou F, Vacher CM, et al. Leptin but not CNTF induces phosphotyrosine phosphatase 1B expression in human neuronal cells (SH-SY5Y): putative explanation of CNTF efficacy in leptin resistant state. Endocrinology 2009; 150: 1182-91.

Berthou F, Rouch C, Gertler A, Gerozissis K, Taouis M. Chronic central leptin infusion differently modulates brain and liver insulin signaling. Mol Cell Endocrinol 2011; 337: 89-95.

Boret SG. Neurodevelopmental actions of leptin. Brain Res 2010; 1350: 2-9.

Figure 2. The role of impaired brain insulin and leptin signaling in Alzheimer's disease. 
Carvalheira JB, Torsoni MA, Ueno $\mathrm{M}$, et al. Cross-talk between the insulin and leptin signaling systems in rat hypothalamus. Obes Res 2005; 13: 48-57.

Cusin I, Zakrzewska KE, Boss O, et al. Chronic central leptin infusion enhances insulinstimulated glucose metabolism and favors the expression of uncoupling proteins. Diabetes 1998; 47: 1014-9.

Elias MF, Elias PK, Sullivan LM, Wolf PA, D'Agostino RB. Obesity diabetes and cognitive deficit: The Framingham Heart Study. Neurobiol Aging 2005; 26: 11-7.

Farris W, Mansourian S, Chang Y, et al. Insulindegrading enzyme regulates the levels of insulin, amyloid beta-protein, and bet-amyloid precursor protein intracellular domain in vivo. Proc Natl Acad USA 2003; 100: 4162-7.

Friedman JM. Leptin, leptin receptors, and the control of body weight. Nutr Rev 1998; 56: 38-6.

Gerozissis K. Brain insulin and feeding: a bidirectional communication. Eur J Pharmacol 490: 59-70, 2004.
Hofbauer KG. Molecular pathways to obesity. Int J Obes 2003; 26: 18-27.

Liu Y, Liu F, Grunke Iqbal I, Iqbal K, Gong CX. Deficient brain insulin signaling pathway in Alzheimer's disease and diabetes. I Pathol 2011; 225: 54-62.

Morrison CD. Leptin signaling in brain: a link between nutrition an cognition? Biochem Biophys Acta 2009; 1792: 401-8.

Niswender KD, Baskin DG, Schwartz MW. Insulin and its evolving partnership with leptin in the hypothalamic control of energy homeostasis. Trends Endocrinol Metab 2004; 15: 362-9.

Ott A, Stolk RP, Hofman A, Van Harskamp F, Grobbee DE, Breteler MM. Association of diabetes mellitus and dementia: the Rotterdam study. Diabetologia 1996; 39: 1392-7.

Paz-Filho G, Wong ML, Licino J. The precognitive effects of leptin in the brain and their clinical implications. Int / Clin Pract 2010; 64: 1808-12.

Pelleymounter MA, Cullen MJ, Baker MB, et al. Effects of the obese gene product on body weight regulation in ob/ob mice. Science 1995; 269: 540-3.

Reaven GM, Thompson LW, Nahum D, Haskins E. Relationship between hyperglycemia and cognitive function in older NIDDM patients. Diabetes Care 1990; 13: 16-21.

Sato N, Takeda S, Uchio-Yamada K, et al. Role of insulin signaling in the interaction between Alzheimer's disease and diabetes mellitus: a missing link to therapeutic potential. Curr Aging Sci 2011; 4: 118-27.

Schwartz MW, Porte Jr D. Diabetes, obesity and the brain. Science 2005; 307: 375-9.

Szanto I, Kahn CR. Selective interaction between leptin and insulin signaling pathway in a hepatic cell line. Proc Natl Acad Sci USA 2000; 97: 2355-60.

Takeda S, Sato N, Uchio-Yamada K. Diabetes accelerated memory dysfunction via cerebrovascular inflammation and abeta deposition in an Alzheimer's mouse model with diabetes. Proc Natl Acad Sci USA 2010; 107: 7036-41. 